\title{
Phase behaviour of DPPC/Lyso-PPC mixtures by spin-label ESR and spectrophotometry
}

\author{
Manuela Pantusa, Luigi Sportelli and Rosa Bartucci* \\ Dipartimento di Fisica, Laboratorio di Biofisica Molecolare and UdR CNISM, Università della \\ Calabria, I-87036 Arcavacata di Rende, CS, Italy
}

\begin{abstract}
The influence of lysopalmitoylphosphatidylcholine (Lyso-PPC) on lamellar dispersions of dipalmitoylphosphatidylcholine (DPPC) has been investigated by spectrophotometry and electron spin resonance (ESR) of spin-labelled phosphatidylcholine at the C-5 or at the C-16 positions in the $s n-2$ acyl chain (5- and 16-PCSL). On increasing the concentration of Lyso-PPC up to $10 \mathrm{~mol} \%$, the spectral anisotropy of 5-PCSL is slightly reduced whereas that of 16-PCSL is markedly increased. This effect of the lysolipid on the DPPC lipid chain mobility is evident from 10 to $50 \mathrm{~mol} \%$ at low temperature in the gel phase, disappears in the fluid phase, is associated with the lack of the pre-transition in the mixed lipid dispersions and it is interpreted as interdigitation of the DPPC lipid chains. At intermediate concentrations and for temperatures in the gel phase, the ESR spectra of both labels in DPPC and Lyso-PPC mixtures are the superposition of an anisotropic lamellar component and of an isotropic micellar signal, suggesting the coexistence of lamellar and micellar aggregates. Chain interdigitation and lamellar-micellar coexistence are not evident for temperature above the melting transition temperature. At high Lyso-PPC content $(\geqslant 60$ mol\%) the mixed lipid dispersions reach the optical clarity, the main transition is no more detected and spin-label ESR spectra typical of micellar dispersions are detected in the whole temperature range investigated.
\end{abstract}

Keywords: Lyso-lipid, DPPC, lamellar phase, micelles, interdigitated chain, spin-label ESR, spectrophotometry

\section{Introduction}

Lysophospholipids are produced from phospholipids and lipoproteins as intermediates in lipid metabolism and turnover [1]. They are found in low amounts in the mammalian cell membrane and in the various membranes of subcellular organelles. Nevertheless, they play a key role both in cellular processes, involving lipid-protein or membrane-membrane interactions [2], and in physiological and pathological processes, including neuronal function, cancer and mechanotransduction $[3,4]$.

Synthetic lysophosphatidylcholines (Lyso-PCs), are wedge shaped single-chain lipids that self assemble in micelles of normal curvature when disperse in buffer above the critical micelle concentration and above the critical micellization temperature [5]. Structurally, Lyso-PCs are compatible with bilayers of saturated symmetrical chain PCs and show a strong affinity for lipid bilayers. However, they induce structural and molecular alterations when inserted in a host lipid model membrane matrix. Indeed, lysophosphatidylcholines, depending on their concentration, when mixed with double chain bilayerforming lipids, affect the lipid chain order and structure of liposomes [6-13], the material property characteristics of lipid bilayers [14], the thermotropic phase behaviour $[8,9,12,15,16]$ and the permeability

\footnotetext{
*Corresponding author. Tel.: +39 0984 496074; Fax: +39 0984 494401; E-mail: bartucci@ fis.unical.it.
} 
properties of lipid vesicles $[7,13,15,16]$. Moreover, at low concentration, they are essential components of thermosensitive liposome used in drug delivery $[15,16]$.

In this context it is of interest to study the molecular interactions and the dynamics of the aggregates that coassemble when lyso-lipids are dispersed together with diacyl bilayer-forming lipids in an aqueous environment. In the present work, we have studied the lyotropic and the thermotropic phase behaviour of fully hydrated binary mixtures of dipalmitoylphosphatidylcholine (DPPC) and Lysopalmitoylphosphatidylcholine (Lyso-PPC) over the entire composition range (0-100 mol\%) and between 10 and $50^{\circ} \mathrm{C}$, a temperature range which encompasses the pre and main lamellar phase transitions of DPPC bilayers [17]. The study has been carried out combining spin-label electron spin resonance (ESR) spectroscopy and spectrophotometry at fixed wavelength. Conventional ESR of chain labelled lipids with the nitroxide reporter group located either at the C-5 position close to polar group region (i.e., 5-PCSL), or at the C-16 position close to the terminal methyl end of the $s n-2$ chain (i.e., 16-PCSL) is used to investigate the segmental lipid chain order and dynamics of the aggregates formed by mixing increasing concentration of Lyso-PPC with DPPC. Additionally, turbidity measurements have been used to monitor the thermotropic phase transitions of the mixed lipid dispersions and to establish phase boundaries.

\section{Materials and methods}

\subsection{Materials}

The synthetic lipids 1,2-dipalmitoyl-sn-glycero-3-phosphocholine and 1-palmitoyl-2-lyso-sn-glycero3-phosphocholine were from Sigma/Aldrich, whereas the spin-labeled lipids 1-palmitoyl-2-(n-(4,4dimethyl-oxazolidine-N-oxyl)stearoyl)-sn-glycero-3-phosphocholine (n-PCSL, $\mathrm{n}=5$ and 16) were from Avanti Polar Lipids. The reagent grade salts for the $10 \mathrm{mM}$ phosphate buffer solution at $\mathrm{pH}=$ 7.2 were from Merck. All materials were used as purchased with no further purification. Bidistilled water was used throughout.

\subsection{Spin-label ESR measurements}

Samples for ESR measurements were prepared by dissolving the required amounts of DPPC and LysoPPC, together with $1 \%$ by weight of the spin-labeled lipid (5- or 16-PCSL), in chloroform. The solvent was evaporated first in a nitrogen gas stream and then under vacuum overnight. The dried lipid samples were fully hydrated with PBS at pH 7.2 (final lipid concentration $25 \mathrm{mM}$ ), by heating and vortexing at $50^{\circ} \mathrm{C}$. The hydrated lipid dispersions were then sealed in a $1 \mathrm{~mm}$ (i.d.) $100 \mu \mathrm{l}$ glass capillaries for ESR measurements and finally incubated for $24 \mathrm{~h}$ at $10^{\circ} \mathrm{C}$ before measuring.

ESR spectra were recorded on a $9 \mathrm{GHz}$ ESP 300 Bruker spectrometer equipped with a Bruker ER $4201 \mathrm{TE}_{102}$ rectangular ESR cavity and a Bruker ER 4111VT variable temperature control unit.

Single-component, low temperature ESR spectra of 5- and 16-PCSL were analyzed by measuring the outer hyperfine splitting, $2 A_{\max }$. The difference between the outer and inner spectral splittings, $\Delta A=2\left(A_{\max }-A_{\min }\right)$, and the width of the high-field hyperfine line, $\Delta H_{-1}$, were instead measured at high temperatures in the spectra of 5- and 16-PCSL, respectively (see Fig. 5). These spectral parameters are sensitive both to the motional amplitude and to the rotational rate of the spin label [18]. The ESR spectra of 5- or 16-PCSL in DPPC/Lyso-PPC dispersions at certain lyso-lipid contents and at intermediate temperatures contain contributions from two distinct motional populations of lipid probes. The composite spectra were resolved into their single components by spectral subtraction as described in $[19,20]$. 


\subsection{Spectrophotometric measurements}

For spectrophotometric measurements, aqueous dispersions of DPPC/Lyso-PPC were prepared as described above for ESR but without addition of spin label. The dried lipid films were first hydrated in PBS at pH 7.5 at a final lipid concentration of $1 \mathrm{mg} / \mathrm{ml}$, and then transferred to 3-ml quartz cells of $1-\mathrm{cm}$ optical path, and finally incubated overnight at $10^{\circ} \mathrm{C}$ before measuring.

Optical density measurements were made at $400 \mathrm{~nm}$ with a Jasco 7850 spectrophotometer equipped with a Peltier thermostatted cell holder (model EHC-441) and a temperature programmer (model TPU-436, accuracy $\pm 0.1^{\circ} \mathrm{C}$ ). A heating rate of $1^{\circ} \mathrm{C} / \mathrm{min}$ was used.

\section{Results and discussion}

\subsection{Spectrophotometry}

The optical density at $400 \mathrm{~nm}\left(\mathrm{OD}_{400}\right)$ of mixtures of DPPC with Lyso-PPC at various molar ratios is given as a function of temperature in Fig. 1. The pre- and the main transitions are clearly seen at temperatures of $T_{\mathrm{p}} \cong 32.3^{\circ} \mathrm{C}$ and $T_{\mathrm{m}} \cong 41.7^{\circ} \mathrm{C}$, respectively, in the thermal profile of DPPC lamellar dispersions (Fig. 1 and Table 1). Addition of the lyso-lipid in the host bilayer matrix of DPPC affects the characteristics of both thermal transitions, the influence being more marked on the pre-transition. Indeed, its amplitude decreases, its width increases, and the pre-transition temperature, $T_{\mathrm{p}}$, decreases progressively to approximately $29^{\circ} \mathrm{C}$ at $6 \mathrm{~mol} \%$ and finally the pre-transition disappears at Lyso-PPC contents $\geqslant 10 \mathrm{~mol} \%$ (Fig. 1 and Table 1). The main transition, instead, is detectable over a broader concentration range. $T_{\mathrm{m}}$ decrease progressively and slowly to approximately $40.2^{\circ} \mathrm{C}$ at $20 \mathrm{~mol} \%$ LysoPPC and then increases reaching at $50 \mathrm{~mol} \%$ a value similar to that observed for DPPC alone.

In agreement with previous studies on DPPC/Lyso-PPC mixtures by differential scanning calorimetry at $2^{\circ} \mathrm{C} / \mathrm{min}[15]$, at $60^{\circ} \mathrm{C} / \mathrm{h}[8,9]$ and at $4^{\circ} \mathrm{C} / \mathrm{h} \mathrm{[12]}$, the characteristics of the main phase transition are slightly different from those of DPPC whereas the melting temperature, $T_{\mathrm{m}}$, is progressively downshifted at most of about $1.5^{\circ} \mathrm{C}$. These shifts are relevant because bring $T_{\mathrm{m}}$ close to the physiological value. For this reason, low amounts of lyso-lipids are used in the formulation of thermosensitive liposomes [15].

Beyond $60 \mathrm{~mol} \%$ of the lyso-lipid, the mixed lipid dispersions are optically clear, the OD values become very low and remain approximately constant throughout the whole temperature range, i.e., the main chain-melting transition is no longer detected as for lyso-PPC micellar dispersions (Fig. 1 and Table 1).

A further feature of Fig. 1 is that the progressive addition of lyso-lipids in the DPPC host lipid matrix affects the optical turbidity at any fixed temperature. As is shown in the inset to Fig. 1 , at $10^{\circ} \mathrm{C}$ the values of $\mathrm{OD}_{400}$ decrease from 2.62 for dispersions of DPPC alone to 2.1 for Lyso-PPC content of $10 \mathrm{~mol} \%$. They remain approximately constant up to $30 \mathrm{~mol} \%$, then they decrease rapidly and progressively to 0.4 at $50 \mathrm{~mol} \%$ Lyso-PPC, and finally drops close to zero for $70 \mathrm{~mol} \%$ onwards.

On the whole, the spectrophotometric measurements indicate a conversion from lamellar phases to micellar aggregates on increasing the content of the lyso-lipid mixed with the diacyl-lipid. Similar phase transformations have been evidenced by spectrophotometry when polymer-lipids are mixed with DPPC $[21,22]$. 


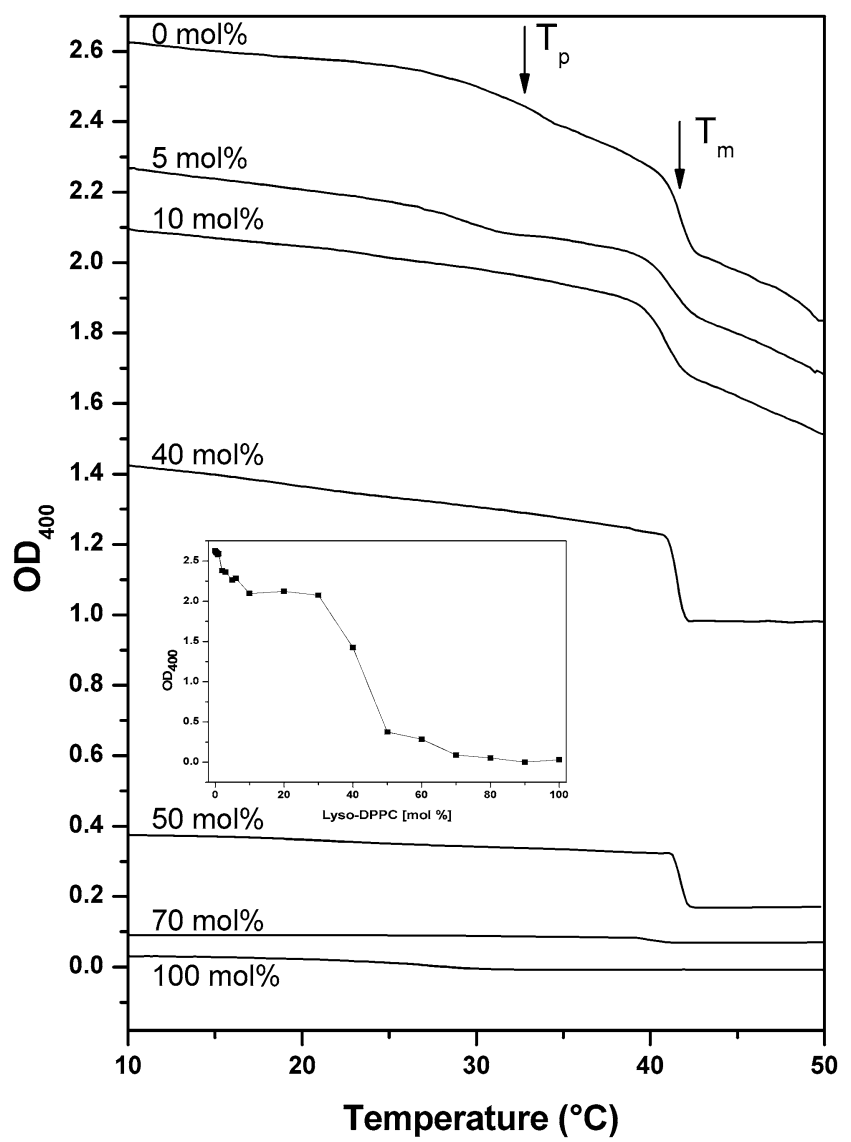

Fig. 1. Temperature dependence of the optical density at $400 \mathrm{~nm}\left(\mathrm{OD}_{400}\right)$ for aqueous mixtures of DPPC/Lyso-PPC. The arrows indicate the temperatures at which the pre- and the main transitions are detected, respectively, in DPPC dispersions. Inset: dependence of $\mathrm{OD}_{400}$ on Lyso-lipid content in aqueous dispersions of DPPC/Lyso-PPC mixtures at $10^{\circ} \mathrm{C}$. The errors are smaller than the symbols.

\subsection{Spin-label electron spin resonance}

The ESR spectra of 5- and 16-PCSL in mixtures of DPPC with different contents of Lyso-PPC at $10^{\circ} \mathrm{C}$ are given in Fig. 2. The spectrum of 5-PCSL in dispersions of DPPC alone is characteristic of spin labels in the slow motional regime on the conventional nitroxide ESR timescale and is typical for phospholipid chains in the $\mathrm{L}_{\beta^{\prime}}$ gel phase [23]. Addition of up to $10 \mathrm{~mol} \%$ of Lyso-PPC in the DPPC dispersions produces small progressive reduction in the anisotropy of the ESR spectra of 5-PCSL. Slight lineshape variations in the central region of the spectra are evident up to $50 \mathrm{~mol} \%$ and an abrupt change in the spectral anisotropy of 5-PCSL occurs only when the Lyso-PPC content reaches $60 \mathrm{~mol} \%$. The spectrum then has an axial lineshape with a considerably smaller hyperfine anisotropy relative to the previous ones. The spectral anisotropy continues to reduce with further addition of lyso-lipids in the lipid mixtures and finally the ESR spectrum of 5-PCSL in dispersions of Lyso-PPC alone at $10^{\circ} \mathrm{C}$ shows spectral features of intermediate mobility characteristic of a micellar phase at low temperature [22,24].

The spectrum of 16-PCSL in dispersions of DPPC in Fig. 2 shows a lower degree of anisotropy (the two outer extreme peaks move in) and a considerable motional broadening (the linewidths are broader) 
Table 1

Temperatures of pre- $\left(T_{\mathrm{p}}\right)$ and main phase transitions $\left(T_{\mathrm{m}}\right)$ of aqueous mixtures of DPPC/Lyso-PPC at different mole fractions, as determined by spectrophotometry

\begin{tabular}{ccc}
\hline Lyso-PPC $(\mathrm{mol} \%)$ & $T_{\mathrm{p}}\left({ }^{\circ} \mathrm{C}\right)^{*}$ & $T_{\mathrm{m}}\left({ }^{\circ} \mathrm{C}\right)^{*}$ \\
\hline 0 & 32.3 & 41.7 \\
1 & 31.3 & 41.5 \\
2 & 31.0 & 41.4 \\
3 & 29.3 & 41.4 \\
5 & 29.2 & 41.2 \\
6 & 29.0 & 41.2 \\
10 & - & 40.7 \\
20 & - & 40.2 \\
30 & - & 40.5 \\
40 & - & 41.2 \\
50 & - & 41.6 \\
60 & - & - \\
70 & - & - \\
80 & - & - \\
90 & - & - \\
100 & - & - \\
\hline
\end{tabular}

${ }^{*}$ The error in $T_{\mathrm{p}}$ and $T_{\mathrm{m}}$ is $0.2^{\circ} \mathrm{C}$.
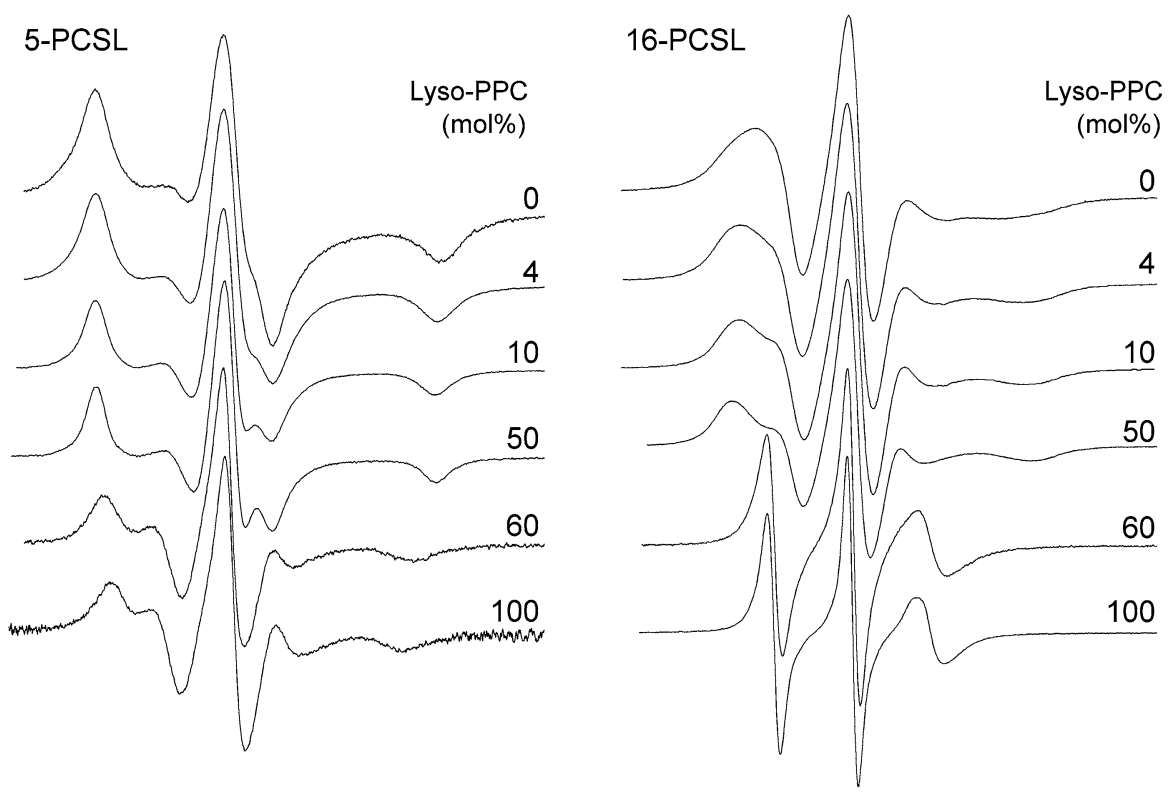

Fig. 2. ESR spectra at $10^{\circ} \mathrm{C}$ of 5-PCSL and 16-PCSL in aqueous mixtures of DPPC/Lyso-PPC at the mole fraction of the lyso-lipid indicated on the figure. Magnetic field sweep width $=100$ Gauss.

relative to that of 5-PCSL in the same system. This spectral difference between the two extreme positions of chain labelling is diagnostic of a lamellar phospholipid gel phase where more motion occurs at the hydrocarbon end [23]. Progressive mixing of Lyso-PPC up to $10 \mathrm{~mol} \%$ with DPPC leads to a considerable 


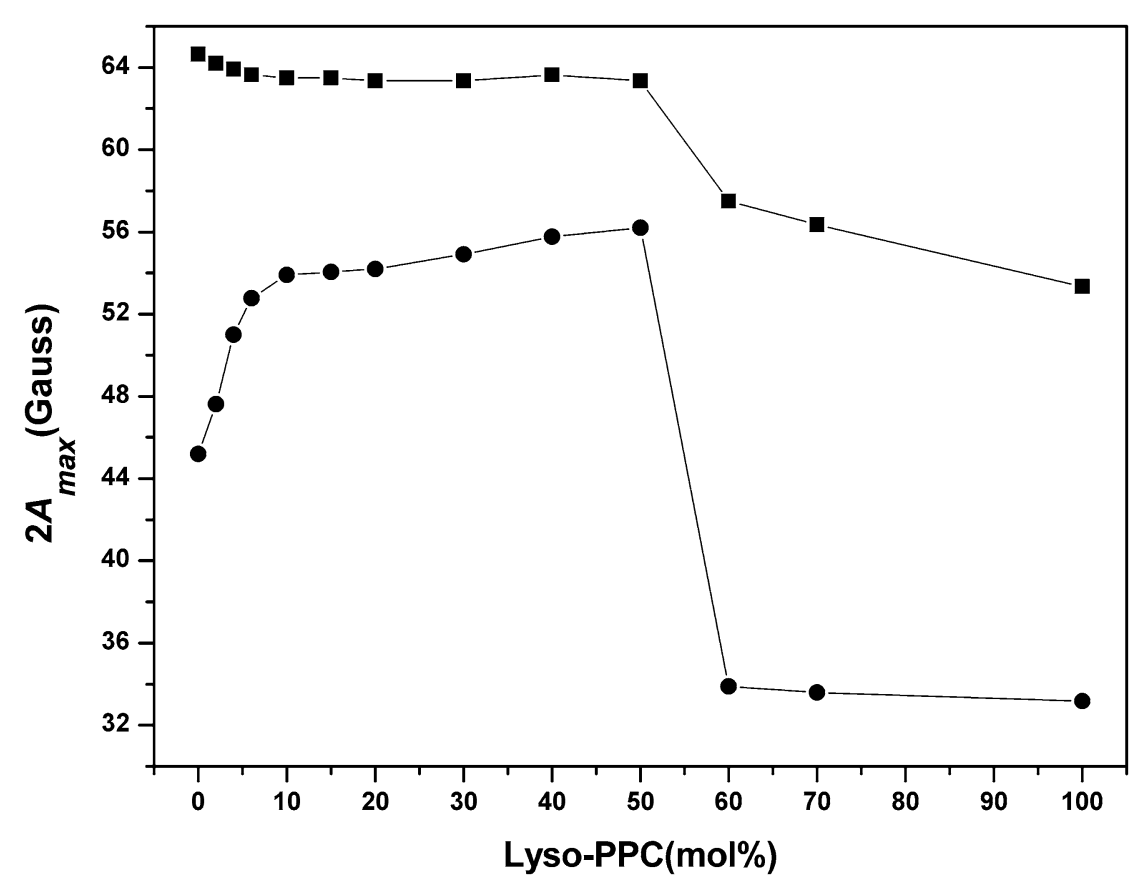

Fig. 3. Dependence on Lyso-PPC content of the outer hyperfine splitting, $2 A_{\max }$, of 5- (squares) and 16-PCSL (circles) in mixed DPPC/Lyso-PPC dispersions at $10^{\circ} \mathrm{C}$. The errors are smaller than the symbols.

increase of the spectral anisotropy which increases slightly further up to $50 \mathrm{~mol} \%$. It comes out that, in this concentration regime, the addition of the lyso-lipids induces motional restriction of 16-PCSL. From $60 \mathrm{~mol} \%$ of Lyso-PPC onwards the spectra of 16-PCSL consist of near-isotropic triplets, with differential broadening of the three hyperfine manifolds and residual anisotropy. Finally, the spectrum of 16-PCSL in dispersions of Lyso-PPC alone at $10^{\circ} \mathrm{C}$ is characteristic of a lipid micelles environment [22,24].

The effects of the lyso-lipid on the acyl chain mobility are clear from the dependence of the outer hyperfine splittings, $2 A_{\max }$, of 5- and 16-PCSL on composition that are given in Fig. 3 at $10^{\circ} \mathrm{C}$. The values of $2 A_{\max }$ for 5-PCSL in DPPC/Lyso-PPC dispersions decrease slightly from 64.64 to 63.5 Gauss on going from 0 to $10 \mathrm{~mol} \%$ Lyso-PPC and then remain approximately constant up to contents of $50 \mathrm{~mol} \%$ of the lyso-lipid. These high values $\left(2 A_{\max }>63\right.$ Gauss $)$ are consistent with expectations for a lamellar gel phase. Following this quasi-constant region, the values of $2 A_{\max }$ decrease rapidly to 57.5 Gauss and to 53.3 Gauss at $60 \mathrm{~mol} \%$ and in micellar dispersions of Lyso-PPC alone, respectively. For 16-PCSL in mixtures of DPPC with Lyso-PPC, the dependence of $2 A_{\max }$ on lipid composition shows first a rapid marked initial increase from 45.2 to 53.9 Gauss between 0 and $10 \mathrm{~mol} \%$, then a further moderate raise to 56.2 at $50 \mathrm{~mol} \%$ and finally flattens off sharply to ca. 33 Gauss at the highest lyso-lipid content.

The above ESR results at $10^{\circ} \mathrm{C}$ give an indication of the types of aggregates formed by adding LysoPPC to DPPC host lipid matrix. Admixture of increasing content of Lyso-PPC in DPPC, slightly enhancing the motional freedom at the beginning of the acyl chain and restricting that at the terminal methyl end, converts the lamellar phase, $\mathrm{L}_{\beta^{\prime}}$, of DPPC in the interdigitated lamellar gel phase, $\mathrm{L}_{\beta \mathrm{I}}$. X-ray diffraction experiments at room temperature in DPPC/Lyso-PPC at 1:1 mol/mol [10] and in DPPC plus $27 \mathrm{~mol} \%$ Lyso-PPC [8,9] clearly indicate fully hydrocarbon chain interdigitation in DPPC induced by the lyso-lipid. Structurally, in an interdigitated phase the hydrocarbon chains from opposing monolayers interpenetrate each other in the gel state. A significant reduction of the bilayer thickness and an increase 
in the area/polarhaed are detected. Calorimetrically, the dispersions do not exhibit the pre-transition and undergo a direct transition from the interdigitated gel state to the fluid lamellar state (for a review, see [25]). The conventional spin-label ESR spectroscopy has been widely used to study a variety of interdigitated lipid systems [23,26-30]. In such systems, the fundamental spectral evidence is the increase of the outermost peak separation of a spin-label having the nitroxide moiety close to the methyl terminus relative to that in noninterdigitated bilayers in the gel state. In same interdigitated bilayers, i.e., DPPC in glycerol or in the presence of ethylene glycol, the motion of the end labelled molecules is quite comparable to that of a spin-label close the polar region in the interdigitated state $[23,26,30]$. In other interdigitated bilayers, i.e., in the interdigitated state induced by ethanol or chaotropic anions, the anisotropy of the end label, although is not increased to the level of that of a label located near the beginning of the chain, is increased relative to that in water and this difference increases on going throughout the gel phase [27,30]. In our case, the evidence for Lyso-PPC induced interdigitation in DPPC is clear from 10 to $50 \mathrm{~mol} \%$. The difference $\Delta 2 A_{\max }=\left(2 A_{\max }(5-\mathrm{PCSL})-2 A_{\max }(16-\mathrm{PCSL})\right)$ is 19.5 Gauss for DPPC in buffer, it reduces to 8.9 Gauss at $10 \mathrm{~mol} \%, 8.4$ Gauss at $30 \mathrm{~mol} \%$ and 7.4 Gauss at $50 \mathrm{~mol} \%$. For comparison, $\Delta 2 A_{\max }$ for stearic acid spin labels is 18 Gauss in buffer, is 10.7 in ethanol and 8.3 Gauss in the presence of $1 \mathrm{M}$ of the chaotropic salt $\mathrm{NaClO}_{4}$. The limited flexibility gradient of increased mobility on proceeding toward the terminal methyl end of the chains normally found in lamellar phases also in the gel phase [23] is, therefore, reduced in the interdigitated lamellar phase of DPPC/Lyso-PPC. The fact that the degree of motional restriction of 16-PCSL does not reach that of 5-PCSL in the interdigitated gel phase of DPPC/Lyso-PPC can be due to a loosened packing density of the acyl chains that characterizes also other interdigitated gel phases (viz. ethanol and chaotropic) $[27,30]$ relative, for instances, to the interdigitated DPPC/glycerol system characterized by a compact structure $[23,26,30]$. The induction of the interdigitated phase in DPPC lipid chains by the lyso-lipid can be rationalized in terms of molecular shape arguments. The wedge shaped Lyso-PPC molecules, on incorporating into DPPC bilayers, may promote an increase in the area per polarhead in the gel phase that is sufficient to induce acyl chain interdigitation in DPPC to minimize the formation of energicatically unfavourable voids in the hydrocarbon region.

The spin-label ESR measurements at $10^{\circ} \mathrm{C}$ also evidence that, at higher lyso-lipid content, the lamellar gel phases are converted into micelles. In micellar aggregates of lyso-lipids the segmental acyl chain order is reduced and the rotational mobility is faster both at the 5th and 16th position along the alkyl chain relative to bilayers of DPPC in the gel $\mathrm{L}_{\beta^{\prime}}$ phase [22,24].

Deeper insight into the phase behaviour of mixtures of DPPC and Lyso-PPC is gained at higher temperatures in the gel phase below the main transition temperature.

The ESR spectra at $37^{\circ} \mathrm{C}$ of 5- and 16-PCSL in dispersions of DPPC, DPPC/Lyso-PPC at 1:1 mol/mol and Lyso-PPC are reported in Fig. 4. As expected, the spectra of the two positional isomers in DPPC dispersions show a considerable temperature-dependent reduction of the spectral anisotropy relative to those recorded for the same systems at $10^{\circ} \mathrm{C}$. Moreover, the spectra of the labels in DPPC alone are asymmetrically broadened. This is due to two unresolved components of slightly different mobility characteristic of the ripple, $\mathrm{P}_{\beta^{\prime}}$, lamellar phase [31]. On increasing the Lyso-PPC content in mixtures with DPPC and for temperatures in the gel phase, the spectra of 5-PCSL show a modest reduction of the anisotropy whereas motional restriction is evident in those of 16-PCSL (data not shown). This behaviour indicates, as expected, the persistence of the interdigitated gel phase. Moreover, at higher Lyso-PPC concentration two component ESR spectra are recorded. As can be seen in Fig. 4, superimposed to a lamellar, anisotropic component (designated $a$ in Fig. 4), a second, isotropic spectral component (designated $i$ in Fig. 4), is clearly resolved in the spectra of 5- and 16-PCSL. This component is contributed 

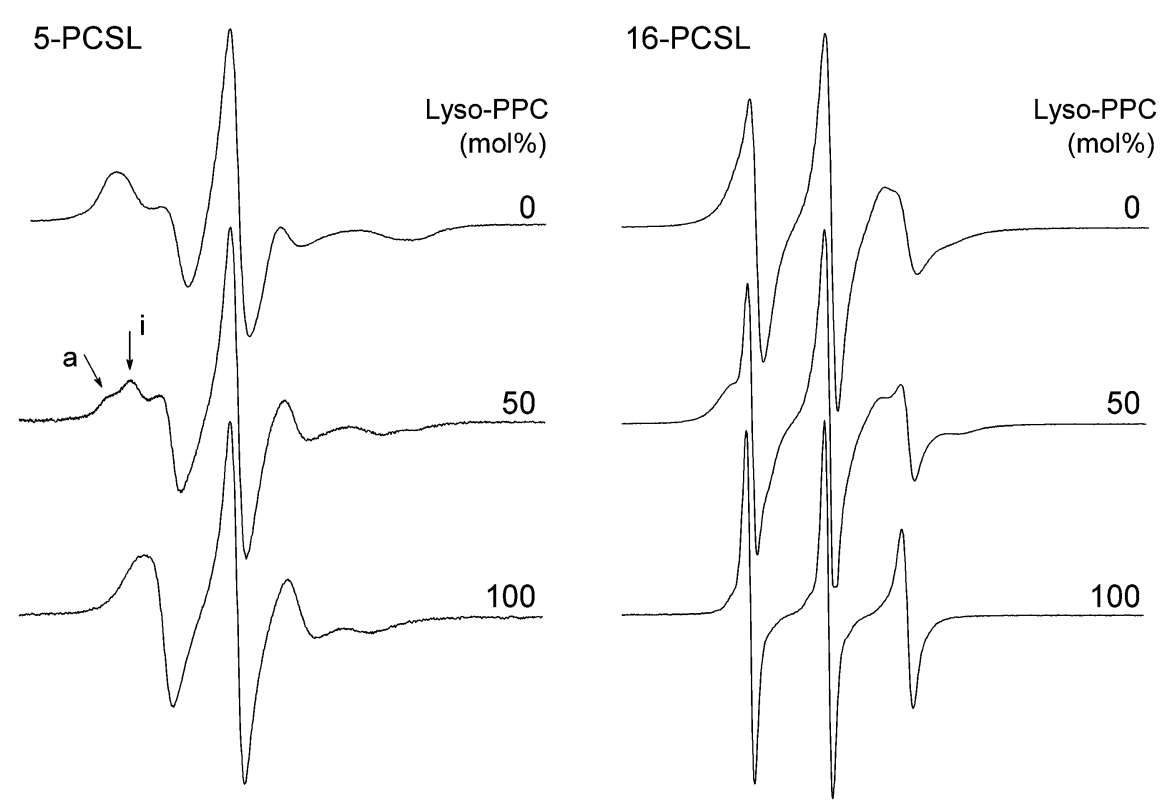

Fig. 4. ESR spectra at $37^{\circ} \mathrm{C}$ of 5-PCSL and 16-PCSL in aqueous mixtures of DPPC/Lyso-PPC at the mole fraction of the lyso-lipid indicated on the figure. Magnetic field sweep width $=100$ Gauss.

by spin-labelled lipids with much higher rotational mobility. Comparison with the ESR spectrum from dispersions of Lyso-PPC alone shows that it corresponds to a population of lipid micelles, because the outer hyperfine splitting is of a very similar size. A coexistence of lamellar and micellar signals has also been evidenced by nuclear magnetic resonance both in uni- and multilamellar vesicles composed of DPPC and Lyso-PPC [6,13].

It is worthy to note that the relative proportion of the micelle-like component increases with increasing content of lyso-lipid and on approaching the chain melting transition temperature of the mixed dispersions. As the lamellar lipid components are in the gel phase whereas the micellar components of the lipid mixture are in a liquid crystalline phase, the two environments can be resolved and quantified using difference spectroscopy $[19,20]$. Subtraction of the micellar component from the two-component spectra of the mixtures, by using as single-component spectrum that of the lyso-lipid alone at an appropriate temperature, yielded the fraction of spin labels in the lamellar, $f$ (lamellar), and in the micellar, $f$ (micelle), phases. The results of spectral subtractions determined from the spectra of 5- and 16-PCSL are in good agreement between them. At $40 \mathrm{~mol} \%$ of Lyso-PPC and at $35^{\circ} \mathrm{C}, f$ (micelle) is ca. 0.2 , it double at $37^{\circ} \mathrm{C}$ and becomes 0.75 at $50 \mathrm{~mol} \%$ and at $37^{\circ} \mathrm{C}$.

From $60 \mathrm{~mol} \%$ onwards of Lyso-PPC, the spectra of both 5- and 16-PCSL are single component typical of micellar dispersions (see spectra in Fig. 5) and show a much lower degree of anisotropy relative to that in the spectra of the corresponding dispersions at $10^{\circ} \mathrm{C}$.

Further interesting features on DPPC/Lyso-PPC phase behaviour are obtained in the fluid phase at $50^{\circ} \mathrm{C}$. The ESR spectra of 5-PCSL and 16-PCSL at $50^{\circ} \mathrm{C}$ in hydrated dispersions of DPPC, DPPC/LysoPPC at $1: 1 \mathrm{~mol} / \mathrm{mol}$ and Lyso-PPC mixtures are given in Fig. 5. The spectra of 5-PCSL are all axial, anisotropically averaged powder patterns that are typical of flexible lipid chains in a fluid liquid-crystalline environment. The extent of motional averaging is, however, in the order: DPPC $\leqslant$ DPPC/Lyso-PPC 1:1 mol/mol « Lyso-PPC, i.e., much larger for the micellar states of the Lyso-lipids than for the fluid lamellar state, $\mathrm{L}_{\alpha}$, of DPPC. The spectra of 16-PCSL are all isotropic ${ }^{14} \mathrm{~N}$-hyperfine 


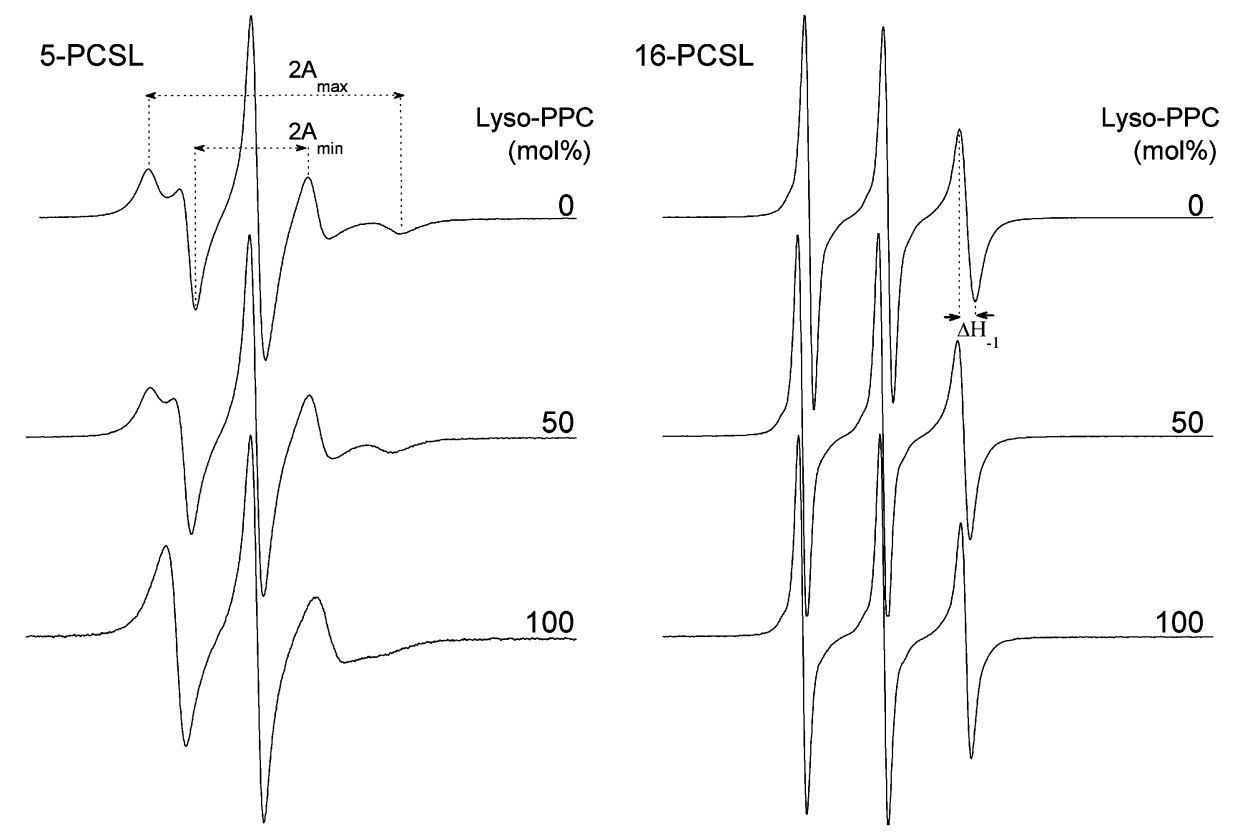

Fig. 5. ESR spectra at $50^{\circ} \mathrm{C}$ of 5-PCSL and 16-PCSL in aqueous mixtures of DPPC/Lyso-PPC at the mole fraction of the lyso-lipid indicated on the figure. Magnetic field sweep width $=100$ Gauss.

triplets. The resonance lines are narrower, however, for the Lyso-lipids than for DPPC, indicating a more rapid segmental chain rotation in the micellar environment.

From the spectra of the two positional isomers in the fluid phase at $50^{\circ} \mathrm{C}$ in DPPC/Lyso-PPC mixed dispersions the following features are singled out: (i) there is no more evidence of DPPC chain interdigitation induced by Lyso-PPC (see spin-label spectra of DPPC/Lyso-PPC mixtures at 1:1 mol/mol in Fig. 5). In contrast to the gel state, there exists, indeed, a much larger degree of anisotropic averaging for the 16-position, relative to the 5-position, of chain labelling. This represents the chain flexibility gradient that is inherent to fluid lamellar liquid crystalline environments [23]. The results also indicate that the influence of the conically shaped Lyso-PPC molecule is primarily on the DPPC chain packing in the gel phase, where the area per headgroup is lower, and it is not appreciable in the fluid phase. (ii) the ESR in DPPC/Lyso-PPC dispersions do not consist of two resolved components, at intermediate content of the lyso-lipid in the mixtures (see spin-label spectra of DPPC/Lyso-PPC at 1:1 mol/mol in Fig. 5). This is because, unlike the situation in the gel phase, the difference in chain motional anisotropy between the fluid lamellar and micellar phases is insufficient for resolution of the two overlapping spectral components.

The lamellar to micelle conversion which occur in DPPC dispersions on adding increasing amounts of Lyso-PPC is also singled out in the fluid phase in the results in Fig. 6. Here, the difference between the outer and inner hyperfine splittings, $\Delta A=2\left(A_{\max }-A_{\min }\right)$, in the spectra of 5-PCSL and the values for the linewidth of the high-field hyperfine manifold, $\Delta H_{-1}$, in the spectra of 16-PCSL in DPPC/LysoPPC mixed dispersions at $50^{\circ} \mathrm{C}$ are given as a function of lipid composition (full squares and circle, respectively). The hyperfine anisotropy, $\Delta A$, decreases first slowly up to $60 \mathrm{~mol} \%$ and then steadily with increasing content of Lyso-lipid in the dispersions. This implies an increasing amplitude of chain rotational motion at high content of lyso-lipid. The trend of $\Delta H_{-1}$ vs. [Lyso-PPC] also reflects the increasing chain segmental mobility on going from bilayers to micelles. 


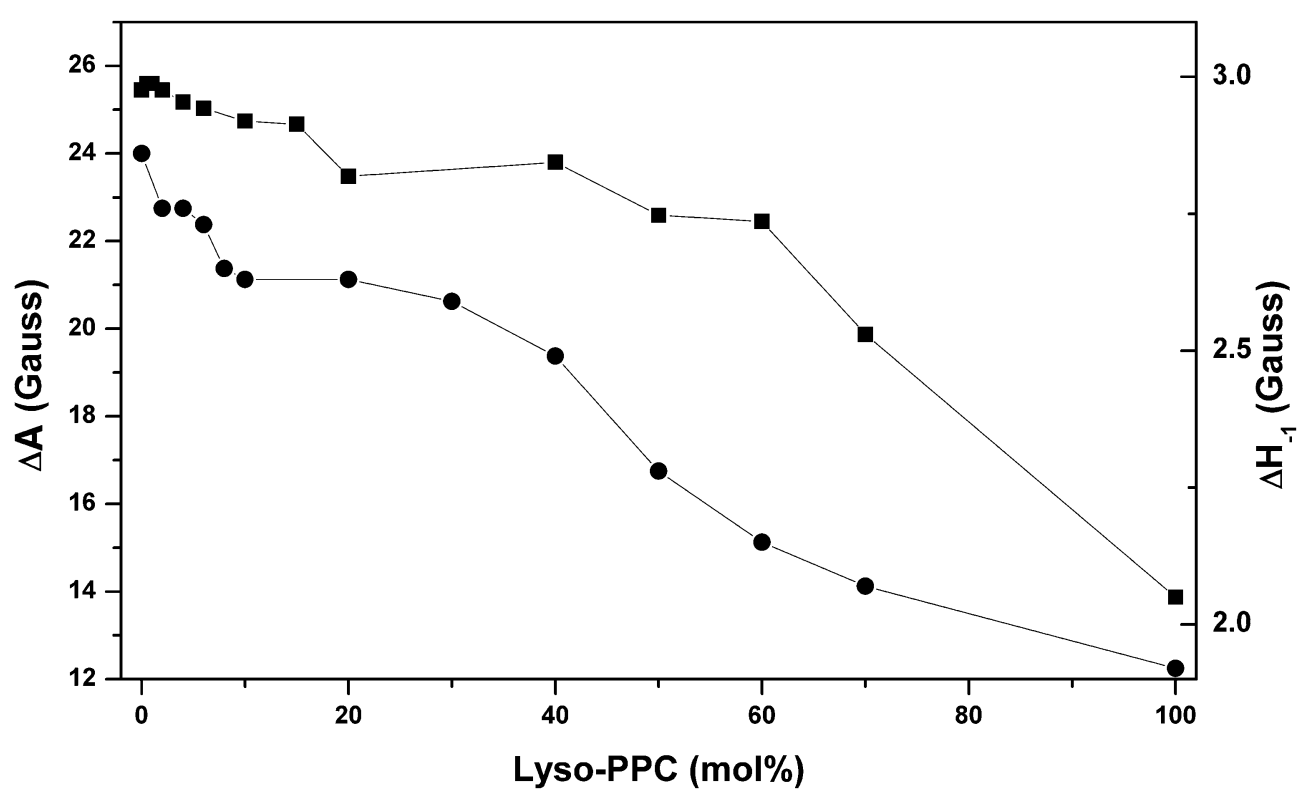

Fig. 6. Dependence on Lyso-PPC content of the difference between the outer and the inner hyperfine splittings, $\Delta A=2\left(A_{\max }-A_{\min }\right)$, of 5-PCSL (squares) and of the peak-to-peak width of the high-field hyperfine line, $\Delta \mathrm{H}_{-1}$, of 16-PCSL (circles) in mixed DPPC/Lyso-PPC dispersions at $50^{\circ} \mathrm{C}$. The errors are smaller than the symbols.

In conclusion, the spectrophotometric and spin-label ESR data reported in the present study provide information on the effects that the incorporation of Lyso-PPC lipids have on the structure of DPPC bilayers and on the phase behaviour of the binary dispersions formed by mixing increasing amount of Lyso-PPC with DPPC. The results evidence a rich polymorphism in binary mixtures of DPPC and LysoPPC at fully hydration. Induction of interdigitated gel lamellar phase at low concentration, coexistence of lamellar and micelle aggregates at intermediate concentration and at intermediate temperatures and micelle formation at high Lyso-PPC content are evidenced.

The effects of Lyso-PPC on the thermotropic and lyotropic phase behaviour of DPPC dispersions, evidenced in this study, may be relevant for their biomedical implications. It is likely that lyso-lipids alter the physical properties of the membranes, that in turn might lead to membrane dysfunction.

\section{Acknowledgments}

M.P. thanks University of Calabria for a research grant.

\section{References}

[1] H. Van den Bosch, Phosphoglyceride metabolism, Annu. Rev. Biochem. 43 (1974), 243-277.

[2] N. Fuller and R. Rand, The influence of lysolipids on the spontaneous curvature and bending elasticity of phospholipid membranes, Biophys. J. 81 (2001), 243-254.

[3] C. Luquain, V. Sciorra and A. Morris, Lysophosphatidic acid signaling: how a small lipid does big things, Trends Biochem. Sci. 28 (2003), 377-383.

[4] H. Ohata, K. Tanaka, N. Maeyama, T. Ikeuchi, A. Kamada, M. Yamanoto and K. Momose, Physiological and pharmacological role of lysophosphatidic acid as modulator in mechanotransduction, Jpn. J. Pharmacol. 87 (2001), 171-176. 
[5] G. Arvidson, I. Brentel, A. Khan, G. Lindblom and K. Fontell, Phase equilibria in four lysophosphatidylcholine/water systems. Exceptional behaviour of 1-palmitoyl-glycero-phosphocholine, Eur. J. Biochem. 152 (1985), 753-759.

[6] S.P. Bhamidipati and J.A. Hamilton, Interactions of Lyso 1-Palmitoylphosphatidylcholine with phospholipids: a ${ }^{13} \mathrm{C}$ and ${ }^{31}$ P-NMR study, Biochemistry 34 (1995), 5666-5677.

[7] Y. Lee and S.I. Chan, Effect of lysolecithin on the structure and permeability of lecithin bilayer vesicles, Biochemistry 16 (1977), 1303-1309.

[8] J.Z. Lu, Y.M. Xu, J.W. Chen and F. Huang, Effect of lysophosphatidylcholine on behaviour and structure of phosphatidylcholine liposomes, Sci. China, Ser. C-Life Sci. 40 (1997), 622-629.

[9] J.Z. Lu, Y.H. Hao and J.W. Chen, Effect of cholesterol on the formation of an interdigitated gel phase in lysophosphatidylcholine and phosphatidylcholine binary mixtures, J. Biochem. 129 (2001), 891-898.

[10] T.J. McIntosh, S. Advani, R.E. Burton, D.V. Zhelev, D. Needham and S.A. Simon, Experimental tests for protrusion and undulation pressures in phospholipids bilayers, Biochemistry 34 (1995), 8520-8532.

[11] K. Mishima, M. Nakajima and T. Ogihara, Effects of lysophospholipids on membrane order of phosphatidylcholine, Coll. Surf. B - Biointerface 33 (2004), 185-189.

[12] M. Pantusa, R. Bartucci and L. Sportelli, Calorimetric and spin-label ESR studies of PEG:2000-DPPE containing DPPC/lyso-PPC mixtures, Coll. Polym. Sci. 285 (2007), 649-656.

[13] C.J.A. Van Echteld, B. De Kruijff, J.G. Mandersloot and J. De Gier, Effects of lysophosphatidylcholines on phosphatidylcholine and phosphatidylcholine/cholesterol liposome systems as revealed by ${ }^{31} \mathrm{P}-\mathrm{NMR}$, electron microscopy and permeability studies, Biochim. Biophys. Acta 649 (1981), 211-220.

[14] D.V. Zhelev, Material property characteristics for lipid bilayers containing lysolipid, Biophys. J. 75 (1988), 321-330.

[15] G. Anyarambhatla and D. Needham, Enhancement of the phase transition permeability of DPPC liposomes by incorporation of MPPC: a new temperature-sensitive liposome for use with mild hyperthermia, J. Liposome Res. 9 (1999), 491-506.

[16] D. Needham, G. Anyarambhatla, G. Kong and M.W. Dewhirst, A new temperature-sensitive liposome for use with mild hyperthermia: characterization and testing in a human tumor xenograft model, Cancer Res. 60 (2000), 1197-1201.

[17] J.R. Silvius, Thermotropic phase transitions of pure lipids in model membranes and their modifications by membrane proteins, in: Lipid-Protein Interaction, Vol. II, P.C. Jost and O.H. Griffith, eds, Wiley, New York, 1982, pp. $239-281$.

[18] D. Marsh, Electron spin resonance: spin labels, in: Membrane Spectroscopy, E. Grell, ed., Springer, Berlin-HeidelbergNew York, 1981, pp. 51-115.

[19] P. Jost and O.H. Griffith, Instrumental aspects of spin labeling, in: Spin Labeling. Theory and Applications, L.J. Berliner, ed., Academic Press, New York, 1976, pp. 251-272.

[20] D. Marsh, Electron spin resonance: spin label probes, in: Techniques in Lipids and Membrane Biochemistry, J.C. Metcalfe and T.R. Hesketh, eds, Elsevier, Amsterdam, 1982, pp. B426/1-B426/44.

[21] S. Belsito, R. Bartucci and L. Sportelli, Sterically stabilized liposomes of DPPC/DPPE-PEG:2000. A spin-label ESR and spectrophotometric study, Biophys. Chem. 75 (1998), 33-43.

[22] S. Belsito, R. Bartucci, G. Montesano, D. Marsh and L. Sportelli, Molecular and mesoscopic properties of hydrophilic polymer-grafted phospholipids mixed with phosphatidylcholine in aqueous dispersion: interaction of dipalmitoyl $N$-poly(ethylene glycol)phosphatidylethanolamine studied by spectrophotometry and spin-label electron spin resonance, Biophys. J. 78 (2000), 1420-1430.

[23] R. Bartucci, T. Pali and D. Marsh, Lipid chain motion in an interdigitated gel phase: conventional and saturation transfer ESR of spin labelled lipids in dipalmitoylphosphatidylcholine-glycerol dispersions, Biochemistry 32 (1993), $274-281$.

[24] R. Bartucci, S. Belsito and L. Sportelli, Spin-label electron spin resonance studies of micellar dispersions of PEGs-PEs polymer-lipids, Chem. Phys. Lipids 124 (2003), 111-122.

[25] J.L. Slater and C.H. Huang, Interdigitated bilayer membranes, Prog. Lipid Res. 27 (1988), 325-359.

[26] J.M. Boggs and G. Rangaraj, Phase transitions and fatty acid spin label behaviour in interdigitated lipid phases induced by glycerol and polymyxin, Biochim. Biophys. Acta 876 (1985), 221-233.

[27] J.M. Boggs, G. Rangaraj and A. Watts, Behaviour of spin-labels in a variety of interdigitated lipid bilayers, Biochim. Biophys. Acta 981 (1989), 243-253.

[28] R. Bartucci and L. Sportelli, Spin label ESR study of the effects of monovalent cations, anions and chaotropics on DPPC multilayers, Biochim. Biophys. Acta 1195 (1994), 229-236.

[29] R. Bartucci, S. Belsito and L. Sportelli, Neutral lipid bilayers interacting with chaotropic anions, Chem. Phys. Lipids 79 (1996), 171-180.

[30] R. Bartucci, G. Montesano and L. Sportelli, Lipid chain mobility in interdigitated DPPC systems, Appl. Magn. Reson. 12 (1997), 41-52.

[31] K. Tushida and I. Hatta, ESR studies of the ripple phase in multilamellar phospholipids bilayers, Biochim. Biophys. Acta 945 (1988), 73-80. 


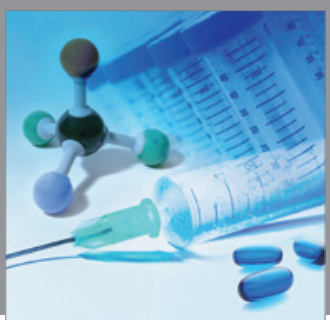

International Journal of

Medicinal Chemistry

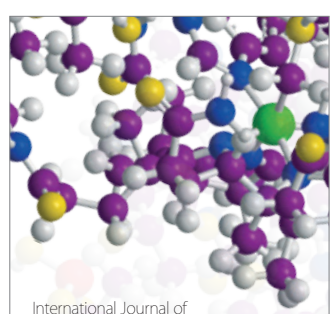

Carbohydrate Chemistry

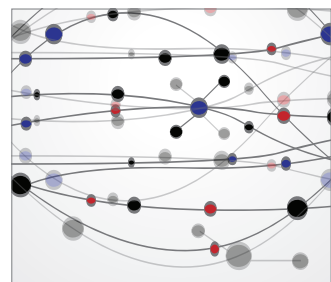

The Scientific World Journal
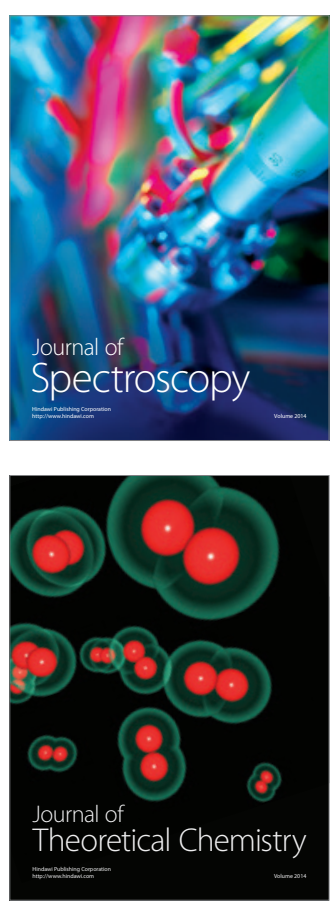
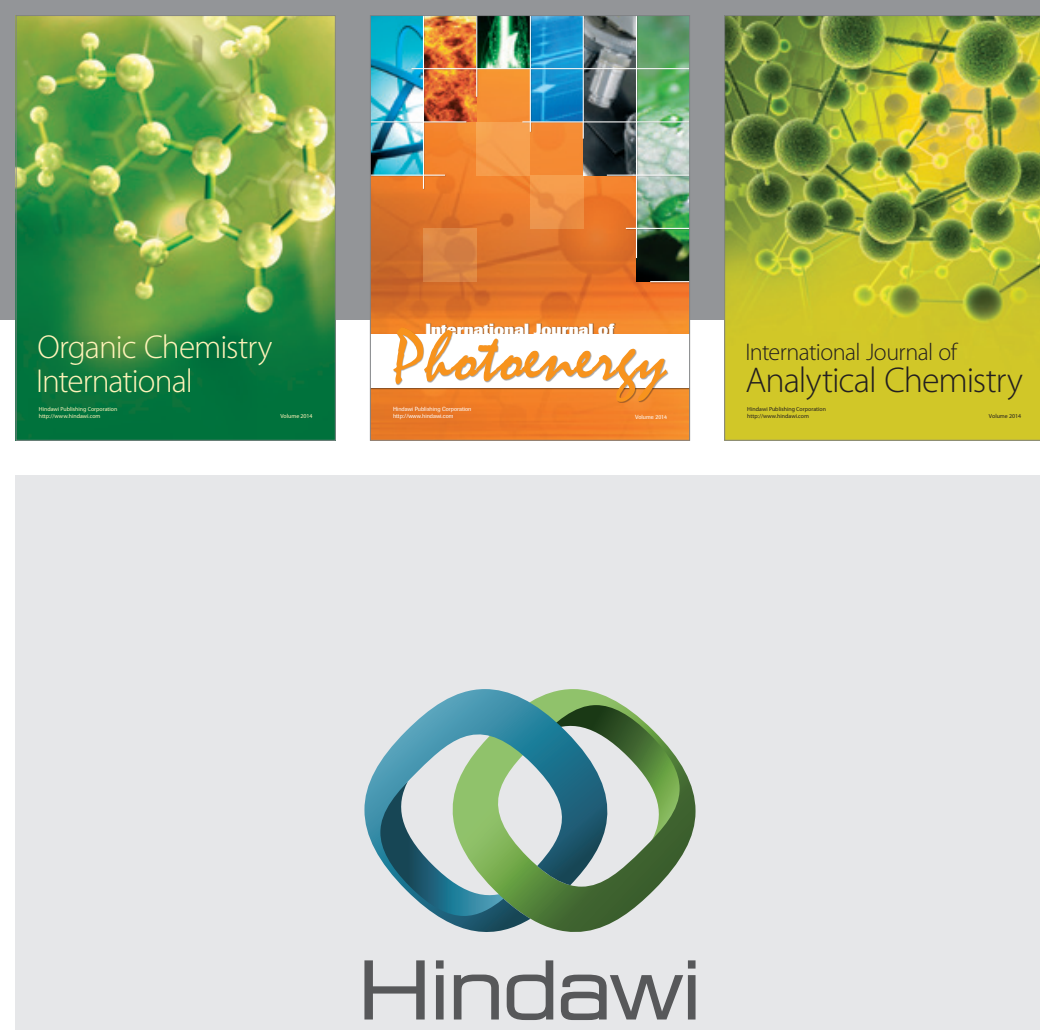

Submit your manuscripts at

http://www.hindawi.com
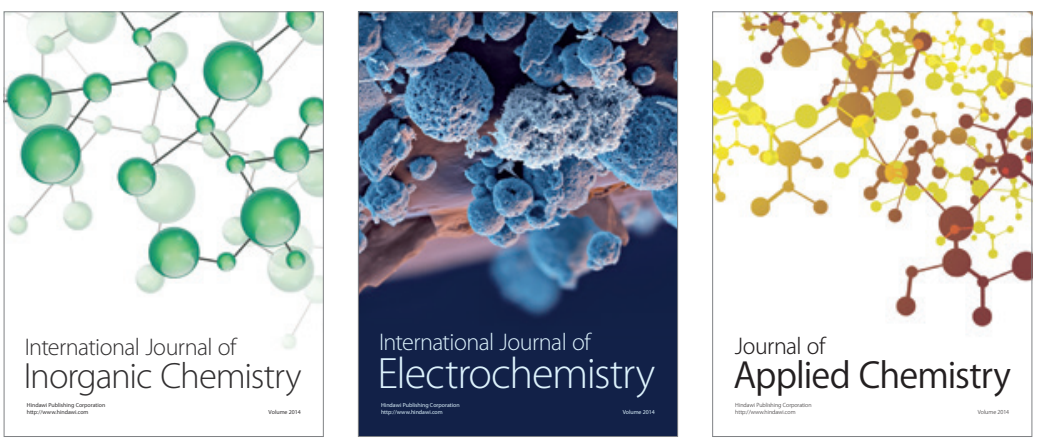

Journal of

Applied Chemistry
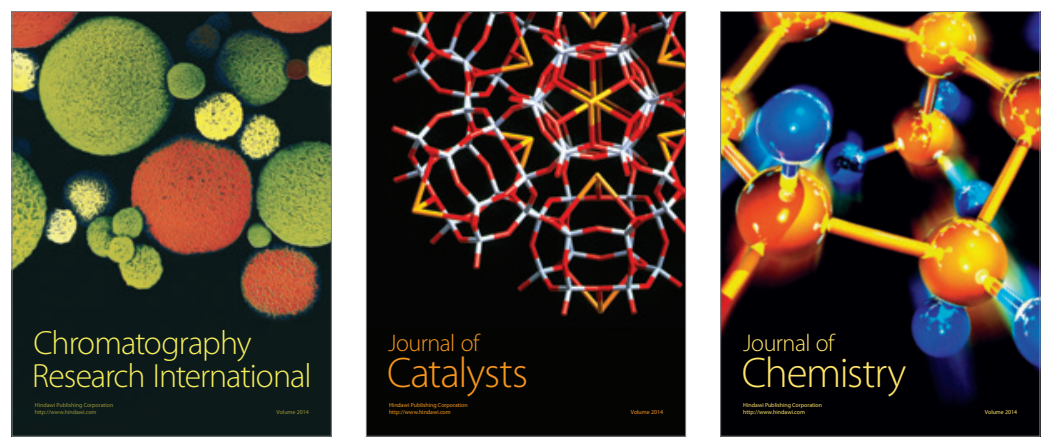
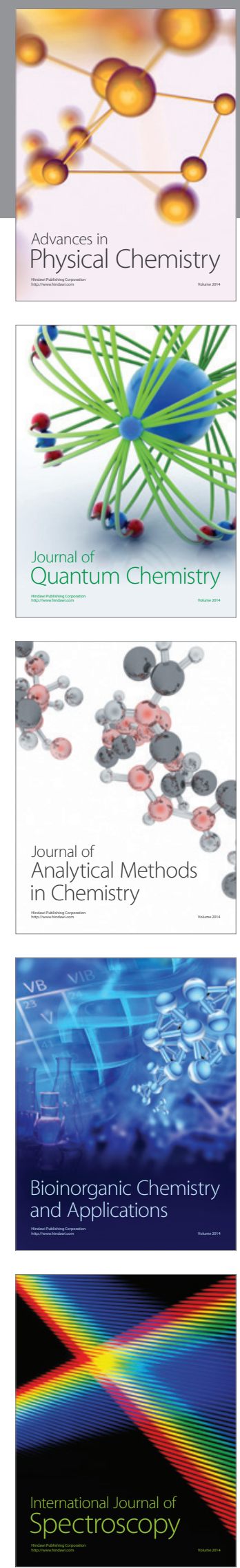